\title{
High-risk retinoblastoma based on age at primary enucleation: a study of 616 eyes
}

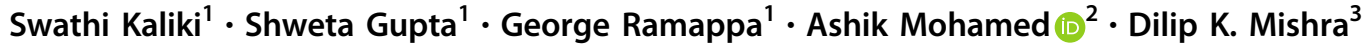

Received: 11 June 2019 / Revised: 17 September 2019 / Accepted: 3 October 2019 / Published online: 25 November 2019

(c) The Author(s), under exclusive licence to The Royal College of Ophthalmologists 2019

\begin{abstract}
Purpose To study the high-risk histopathology features of retinoblastoma based on age at primary enucleation. Methods Retrospective study of 616 patients.

Results The mean age at presentation and primary enucleation for retinoblastoma was 34 months (median, 28 months; range, $<1-455$ months). Of these cases, $128(21 \%)$ were aged $\leq 1$ year, $149(24 \%)$ were in the age group of $1-2$ years, $117(19 \%)$ in 2-3 years, $104(17 \%)$ in 3-4 years, and $118(19 \%)$ were $>4$ years of age at the time of enucleation. Bilateral retinoblastoma $(34 \% ; p<0.0001)$ and buphthalmos $(20 \% ; p<0.0001)$ were more common in children $\leq 1$ year of age. Anterior chamber pseudohypopyon $(15 \% ; p<0.0001)$ and vitreous seeds $(53 \% ; p<0.0001)$ were more common in children aged $>4$ years. Based on 8th edition American Joint Committee on Cancer staging system, pT3 was less common in children $\leq 1$ year of age $(13 \% ; p<0.001)$. Based on histopathology, $38 \%$ patients had high-risk features including $24 \%$ children aged $\leq 1$ year, $42 \%$ in the age group of 1-2 years, $34 \%$ in 2-3 years age group, $45 \%$ in 3-4 years age group, and $48 \%$ patients were $>4$ years of age. Post-laminar optic nerve infiltration $(6 \% ; p=0.02)$ and massive choroidal infiltration $(9 \% ; p=0.04)$ was least common in children $\leq 1$ year of age. Over a mean follow-up period of 52 months (median, 36 months; range, $<1-218$ months), systemic metastasis and death occurred in $9 \%$ patients despite adjuvant systemic chemotherapy.

Conclusion The predominant high-risk histopathology feature of retinoblastoma varies with age at primary enucleation.
\end{abstract}

\section{Introduction}

Enucleation for retinoblastoma (RB) is a common modality of treatment in the developing countries owing to advanced intraocular disease at presentation. It forms the primary modality of treatment in nearly $35 \%$ patients with $\mathrm{RB}$ in India [1]. The definition of high-risk RB is not uniform across the oncology groups, though massive choroidal infiltration, post-laminar optic nerve infiltration, scleral and extrascleral tumour infiltration are considered as the highrisk histopathology features by most groups [2-6]. The

Swathi Kaliki

kalikiswathi@yahoo.com

1 The Operation Eyesight Universal Institute for Eye Cancer, L V Prasad Eye Institute, Hyderabad 500034, India

2 Ophthalmic Biophysics (AM), L V Prasad Eye Institute, Hyderabad 500034, India

3 Ophthalmic Pathology Service (DKM), L V Prasad Eye Institute, Hyderabad 500034, India occurrence of high-risk RB is twofold more common in Asian Indians with threefold increased risk of massive choroidal infiltration and fivefold increased risk of optic nerve infiltration compared with the West [7].

The age at detection of RB may vary in the developing and developed countries. However, early age at diagnosis of RB does not guarantee that the tumour is at an early stage and all eyes can be saved, though the proportion of patients needing enucleation may vary with age and stage of disease at presentation. Enucleation may be needed even in neonates with advanced intraocular RB $[8,9]$. Based on age at presentation, the clinical signs and symptoms vary in cases with RB [8-11]. We hypothesize that the high-risk histopathological features of RB may also vary based on age at enucleation. In this study, we explore the differences in the high-risk histopathological features of RB based on age at primary enucleation.

\section{Methods}

This retrospective study was conducted at the Operation Eyesight Universal Institute for Eye Cancer, L V Prasad 
Eye Institute, Hyderabad, India. Institutional Review Board of L V Prasad Eye Institute, Hyderabad approved this study. All RB cases that underwent enucleation between the years January 2000 and June 2016 were reviewed. The patients who underwent primary enucleation for RB and had adequate clinical and histopathology data were included in this study. Those with inadequate data or had undergone secondary enucleation for RB were excluded.

Age at presentation, gender, hereditary pattern, tumour laterality, presenting complaints, and duration of symptoms were noted. The recorded clinical features included horizontal corneal diameter, intraocular pressure, anterior chamber findings (anterior chamber seeds/pseudohypopyon or hyphema), iris details (iris neovascularization, corectopia, posterior synechiae, ectropion uveae), lens status, evidence of orbital pseudocellulitis, and fundus details (tumour basal diameter, tumour thickness, subretinal fluid, subretinal or vitreous seeds, vitreous haemorrhage). Based on clinical features, the tumours were classified according to the Reese-Ellsworth Classification [12], International Classification of Retinoblastoma (ICRB) [13], and the 8th edition of American Joint Committee on Cancer (AJCC) staging system [14]. B-scan ultrasonography and orbital imaging (computed tomography or magnetic resonance imaging) findings were recorded. The details of treatment were recorded. The reason for enucleation was noted. In bilateral cases, the worse eye was enucleated as appropriate.

The following histopathology features were recorded: growth pattern (endophytic, exophytic, mixed endophyticexophytic, diffuse infiltrating RB), tumour differentiation (well-differentiated, moderately differentiated, poorly differentiated, undifferentiated), anterior chamber seeding, iris infiltration, ciliary body infiltration, choroidal invasion [minor $(<3 \mathrm{~mm}$ in greatest dimension) or major $(>3 \mathrm{~mm}$ in greatest dimension of a single lesion or sum of all areas of choroidal invasion)], optic nerve infiltration (pre-laminar, laminar, post-laminar, or optic nerve transection), scleral infiltration, or microscopic extrascleral infiltration.

High-risk RB was defined as the presence of tumour seeds in the anterior chamber, iris/ciliary body infiltration, massive $(\geq 3 \mathrm{~mm})$ choroidal invasion, post-laminar optic nerve infiltration, optic nerve transection, combined prelaminar/laminar optic nerve infiltration and minor choroidal invasion, or scleral/extrascleral tumour infiltration. All patients with high-risk RB were advised adjuvant systemic intravenous chemotherapy and those with involvement of optic nerve transection or scleral/ extrascleral tumour infiltration were also advised additional external beam radiotherapy to the orbit. The details of adjuvant treatment, events of metastasis, and death were noted.

\section{Statistical methods}

The statistical analysis was performed using the software Origin v7.0 (OriginLab Corporation, Northampton, USA). The continuous data were summarized in mean, median and range, and categorical data in proportions. The continuous data were checked for normality by Shapiro-Wilk test and for equality of variance by Levene test. The continuous and categorical data were compared among various age groups by Kruskal-Wallis test and Chi-square test, respectively, and, for pair-wise comparisons, by Mann-Whitney test and Chi-square test respectively. A $p$ value of $<0.05$ was considered statistically significant. For multiple pair-wise comparisons, Bonferroni correction was applied and $p$ value of $<0.0125$ was considered statistically significant.

\section{Results}

A total of 916 patients underwent enucleation for RB during the study period. Of these, 288 patients underwent secondary enucleation and 12 patients had inadequate data and were excluded from the study. A total of 616 patients who underwent primary enucleation for RB were included in the study. Of these cases, $128(21 \%)$ were aged $\leq 1$ year, 149 (24\%) were in the age group of 1-2 years, 117 (19\%) in 2-3 years, $104(17 \%)$ in $3-4$ years, and $118(19 \%)$ were $>4$ years of age at the time of enucleation.

The demographic and clinical features in Table 1. The mean age at presentation was 34 months (median, 28 months; range, $<1-455$ months). The mean duration of symptoms was 4 months (median, 1 month; range, $<1-78$ months). The time interval between the date of presentation and date of primary enucleation was less than 2 weeks in all cases. Of all cases, 57\% patients were males and $43 \%$ were females. Bilateral RB $(34 \% ; p<0.0001)$ and buphthalmos $(20 \% ; p<0.0001)$ were more common in children $\leq 1$ year of age. Anterior chamber pseudohypopyon $(15 \% ; p<0.0001)$ and vitreous seeds $(53 \% ; p<0.0001)$ were more common in children aged $>4$ years. Vitreous seeds $(21 \%)$ were less common in children $\leq 1$ year of age.

Based on AJCC (Table 2), pT3 was less common in children $\leq 1$ year of age $(13 \% ; p<0.001)$. Based on histopathology (Table 3), well-differentiated tumours were more common in children $\leq 1$ year of age $(56 \% ; p<0.001)$, and poorly differentiated tumours were commoner in children $>3$ years of age $(65 \% ; p<0.001)$. Of all patients, $38 \%$ had high-risk features including $24 \%$ children aged $\leq 1$ year, $42 \%$ in the age group of $1-2$ years, $34 \%$ in $2-3$ years age group, $45 \%$ in 3-4 years age group, and $48 \%$ patients were $>4$ years of age. The occurrence of high-risk histopathology features $(p=0.06)$ and the number of high-risk histopathology features $(p=0.85)$ were not statistically 
Table 1 High-risk retinoblastoma based on age at presentation: demographics and clinical features

\begin{tabular}{|c|c|c|c|c|c|c|c|}
\hline Feature & $\begin{array}{l}\text { All ages } \\
(n=616)\end{array}$ & $\begin{array}{l}\text { Age } \leq 1 \text { year } \\
(n=128)\end{array}$ & $\begin{array}{l}\text { Age } 1-2 \text { years } \\
(n=149)\end{array}$ & $\begin{array}{l}\text { Age } 2-3 \text { years } \\
(n=117)\end{array}$ & $\begin{array}{l}\text { Age } 3-4 \text { years } \\
(n=104)\end{array}$ & $\begin{array}{l}\text { Age }>4 \text { years } \\
(n=118)\end{array}$ & $p$ value \\
\hline $\begin{array}{l}\text { Age at presentation } \\
\text { (months) mean } \\
\text { (median, range) }\end{array}$ & $34(28,<1-455)$ & $8(9,<1-12)$ & $20(20,13-24)$ & $31(32,25-36)$ & $43(43,37-48)$ & $73(62,49-455)$ & N/A \\
\hline \multicolumn{8}{|l|}{ Gender } \\
\hline Male & $352(57)$ & $79(62)$ & $80(54)$ & $67(57)$ & $56(54)$ & $70(59)$ & 0.95 \\
\hline Female & $264(43)$ & $49(38)$ & $69(46)$ & $50(43)$ & $48(46)$ & $48(41)$ & 0.91 \\
\hline \multicolumn{8}{|l|}{ Hereditary pattern } \\
\hline Sporadic & 608 (99) & $125(98)$ & 147 (99) & $117(100)$ & $103(99)$ & $116(98)$ & 1.00 \\
\hline Familial & $8(1)$ & $3(2)$ & $2(1)$ & $0(0)$ & $1(1)$ & $2(2)$ & 0.59 \\
\hline \multicolumn{8}{|l|}{ Laterality } \\
\hline Unilateral & $516(84)$ & $85(66)$ & $126(85)$ & $108(92)$ & $96(92)$ & $101(86)$ & 0.43 \\
\hline Bilateral & $100(16)$ & $43(34)$ & $23(5)$ & $9(8)$ & $8(8)$ & $17(14)$ & $<0.0001^{\mathrm{a}}$ \\
\hline $\begin{array}{l}\text { Duration of symptoms } \\
\text { (months) mean } \\
\text { (median, range) }\end{array}$ & $4(1,<1-78)$ & $3(1,<1-44)$ & $4(2,<1-27)$ & $4(2,<1-36)$ & $5(1,<1-48)$ & $4(1,<1-78)$ & 0.20 \\
\hline$<6$ months & $539(88)$ & $118(92)$ & $129(87)$ & $99(85)$ & $87(84)$ & $106(90)$ & 0.99 \\
\hline$>6$ months & $77(12)$ & $10(8)$ & $20(13)$ & $18(15)$ & 17 (16) & $12(10)$ & 0.36 \\
\hline Buphthalmos & $55(9)$ & $26(20)$ & $11(7)$ & $6(5)$ & 7 (7) & $5(4)$ & $<0.0001^{b}$ \\
\hline Secondary glaucoma & $205(33)$ & $35(27)$ & $45(30)$ & $35(30)$ & $44(42)$ & $46(39)$ & 0.37 \\
\hline $\begin{array}{l}\text { Anterior chamber seeds/ } \\
\text { pseudohypopyon }\end{array}$ & $35(6)$ & $3(2)$ & $5(3)$ & $3(3)$ & $6(6)$ & $18(15)$ & $<0.0001^{\mathrm{c}}$ \\
\hline Iris neovascularization & $197(32)$ & $29(23)$ & $46(31)$ & $35(30)$ & $40(38)$ & $47(40)$ & 0.23 \\
\hline Hyphema & $37(6)$ & $6(5)$ & $12(8)$ & $6(5)$ & $6(6)$ & $7(6)$ & 0.83 \\
\hline Ectropion uveae & $133(22)$ & $23(18)$ & $30(20)$ & $23(20)$ & $26(25)$ & $31(26)$ & 0.67 \\
\hline Cataract & $29(5)$ & $2(2)$ & $8(5)$ & $5(4)$ & $8(8)$ & $6(5)$ & 0.32 \\
\hline $\begin{array}{l}\text { Largest tumour base }(\mathrm{mm}) \\
\text { mean (median, range) }\end{array}$ & $19(19,5-24)$ & $19(20,10-24)$ & $18(19,5-24)$ & $19(19,10-24)$ & $18(18,6-24)$ & $18(18,8-24)$ & 0.30 \\
\hline $\begin{array}{l}\text { Tumour thickness }(\mathrm{mm}) \\
\text { mean (median, range) }\end{array}$ & $15(15,3-24)$ & $16(16,4-22)$ & $16(16,3-23)$ & $15(16,8-24)$ & $15(15,3-22)$ & $15(15,6-22)$ & 0.38 \\
\hline Subretinal fluid & $265(43)$ & $70(55)$ & $69(46)$ & $48(41)$ & $36(35)$ & $42(36)$ & 0.26 \\
\hline Subretinal seeds & $130(21)$ & $28(22)$ & $30(20)$ & $27(23)$ & $24(23)$ & $21(18)$ & 0.92 \\
\hline Vitreous seeds & $241(39)$ & $27(21)$ & $56(38)$ & $60(51)$ & $35(34)$ & $63(53)$ & $0.003^{\mathrm{d}}$ \\
\hline Vitreous haemorrhage & $67(11)$ & $8(6)$ & 17 (11) & $8(7)$ & $16(15)$ & $18(15)$ & 0.11 \\
\hline
\end{tabular}

N/A not applicable

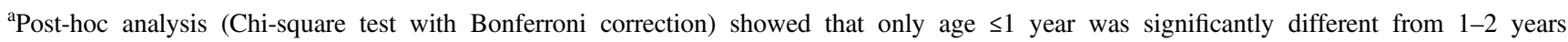
$(p=0.006), 2-3$ years $(p<0.001), 3-4$ years $(p<0.001)$ and $>4$ years $(p=0.006)$

${ }^{\mathrm{b}}$ Post-hoc analysis (Chi-square test with Bonferroni correction) showed that only age $\leq 1$ year was significantly different from $1-2$ years $(p=0.006), 2-3$ years $(p=0.002), 3-4$ years $(p=0.01)$ and $>4$ years $(p=0.001)$

${ }^{c}$ Post-hoc analysis (Chi-square test with Bonferroni correction) showed that only ages $>4$ years was significantly different from $\leq 1$ year $(p=0.001), 1-2$ years $(p=0.002)$ and $2-3$ years $(p=0.002)$

${ }^{\mathrm{d}}$ Post-hoc analysis (Chi-square test with Bonferroni correction) showed that only age $\leq 1$ year was significantly different from $2-3$ years $(p=0.001)$ and $>4$ years $(p<0.0001)$

significant based on age at enucleation. Overall, postlaminar optic nerve infiltration was common in children at $1-2$ years of age (23\%), massive choroidal infiltration at 3-4 years (28\%), and anterior chamber tumour seeding, iris infiltration, and ciliary body infiltration in children $>4$ years of age $(14 \%, 9 \%$, and $9 \%$, respectively). Poorly differentiated tumours $(15 \% ; p<0.001)$, massive choroidal invasion $(9 \% ; p=0.04)$ and post-laminar optic nerve infiltration $(6 \% ; p=0.02)$ were less common in children $\leq 1$ year of age when compared with other age groups.

Of 237 patients with high-risk RB, 226 (95\%) received adjuvant chemotherapy (Table 4). The remaining 11 patients did not receive adjuvant chemotherapy and were lost to follow-up. Overall, systemic metastasis and death 
Table 2 High-risk retinoblastoma based on age at presentation: Tumour classification

\begin{tabular}{|c|c|c|c|c|c|c|c|}
\hline Feature & $\begin{array}{l}\text { All ages } \\
(n=616)\end{array}$ & $\begin{array}{l}\text { Age }<1 \text { year } \\
(n=128)\end{array}$ & $\begin{array}{l}\text { Age } 1-2 \text { years } \\
(n=149)\end{array}$ & $\begin{array}{l}\text { Age } 2-3 \text { years } \\
(n=117)\end{array}$ & $\begin{array}{l}\text { Age } 3-4 \text { years } \\
(n=104)\end{array}$ & $\begin{array}{l}\text { Age }>4 \text { years } \\
(n=118)\end{array}$ & $p$ value \\
\hline \multicolumn{8}{|c|}{ ICRB classification } \\
\hline Group A & $0(0)$ & $0(0)$ & $0(0)$ & $0(0)$ & $0(0)$ & $0(0)$ & N/A \\
\hline Group B & $0(0)$ & $0(0)$ & $0(0)$ & $0(0)$ & $0(0)$ & $0(0)$ & N/A \\
\hline Group C & $0(0)$ & $0(0)$ & $0(0)$ & $0(0)$ & $0(0)$ & $0(0)$ & N/A \\
\hline Group D & $71(12)$ & $14(11)$ & $15(10)$ & $13(11)$ & $13(13)$ & $16(14)$ & 0.95 \\
\hline Group E & $545(88)$ & $114(89)$ & $134(90)$ & $104(89)$ & $91(88)$ & $102(86)$ & 1.00 \\
\hline \multicolumn{8}{|c|}{ Reese-Ellsworth classification } \\
\hline $1 \mathrm{~A}$ & $0(0)$ & $0(0)$ & $0(0)$ & $0(0)$ & $0(0)$ & $0(0)$ & N/A \\
\hline $1 \mathrm{~B}$ & $0(0)$ & $0(0)$ & $0(0)$ & $0(0)$ & $0(0)$ & $0(0)$ & N/A \\
\hline $2 \mathrm{~A}$ & $0(0)$ & $0(0)$ & $0(0)$ & $0(0)$ & $0(0)$ & $0(0)$ & N/A \\
\hline $2 \mathrm{~B}$ & $0(0)$ & $0(0)$ & $0(0)$ & $0(0)$ & $0(0)$ & $0(0)$ & N/A \\
\hline $3 \mathrm{~A}$ & $0(0)$ & $0(0)$ & $0(0)$ & $0(0)$ & $0(0)$ & $0(0)$ & N/A \\
\hline $3 \mathrm{~B}$ & $0(0)$ & $0(0)$ & $0(0)$ & $0(0)$ & $0(0)$ & $0(0)$ & N/A \\
\hline $4 \mathrm{~A}$ & $2(<1)$ & $1(<1)$ & $1(<1)$ & $0(0)$ & $0(0)$ & $0(0)$ & 0.65 \\
\hline $4 \mathrm{~B}$ & $1(<1)$ & $0(0)$ & $0(0)$ & $0(0)$ & $0(0)$ & $1(1)$ & 0.38 \\
\hline $5 \mathrm{~A}$ & $272(44)$ & $82(64)$ & $66(44)$ & $42(36)$ & $49(47)$ & $33(28)$ & $0.007^{\mathrm{a}}$ \\
\hline $5 B$ & $341(55)$ & $45(35)$ & $82(55)$ & $75(64)$ & $55(53)$ & $84(71)$ & $0.03^{\mathrm{b}}$ \\
\hline \multicolumn{8}{|c|}{ 8th edition cT AJCC classification } \\
\hline $\mathrm{cT} 1$ & $0(0)$ & $0(0)$ & $0(0)$ & $0(0)$ & $0(0)$ & $0(0)$ & N/A \\
\hline $\mathrm{cT} 2$ & $30(5)$ & $5(4)$ & $7(5)$ & $5(4)$ & 7 (7) & $6(5)$ & 0.91 \\
\hline cT3 & $586(95)$ & $123(96)$ & $142(95)$ & $112(96)$ & $97(93)$ & $112(95)$ & 1.00 \\
\hline cT4 & $0(0)$ & $0(0)$ & $0(0)$ & $0(0)$ & $0(0)$ & $0(0)$ & N/A \\
\hline \multicolumn{8}{|c|}{ 8th edition pT AJCC classification } \\
\hline pT1 & $262(43)$ & $64(50)$ & $61(41)$ & $53(45)$ & $42(40)$ & $42(36)$ & 0.66 \\
\hline pT2 & $167(27)$ & $47(37)$ & $31(21)$ & $30(26)$ & $21(20)$ & $38(32)$ & 0.11 \\
\hline pT3 & $171(28)$ & $16(13)$ & $53(36)$ & $29(25)$ & $38(37)$ & $35(30)$ & $<0.001^{\mathrm{c}}$ \\
\hline pT4 & $16(3)$ & $1(<1)$ & $4(3)$ & $5(4)$ & $3(3)$ & $3(3)$ & 0.58 \\
\hline
\end{tabular}

$I C R B$ international classification of retinoblastoma, $c T$ clinical tumour, $p T$ pathological tumour, AJCC American Joint Committee Classification, N/A not applicable

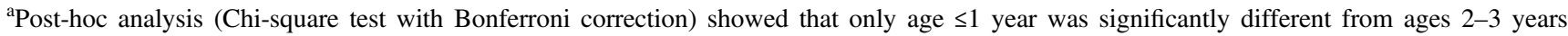
$(p=0.011)$ and ages $>4$ years $(p=0.001)$

${ }^{b}$ Post-hoc analysis (Chi-square test with Bonferroni correction) showed that only age $\leq 1$ year was significantly different from ages $2-3$ years $(p=0.008)$ and ages $>4$ years $(p=0.002)$

${ }^{c}$ Post-hoc analysis (Chi-square test with Bonferroni correction) showed that only age $\leq 1$ year was significantly different from $1-2$ years $(p=0.001), 3-4$ years $(p=0.001)$ and $>4$ years $(p=0.007)$

occurred in $22(4 \%)$ patients over a mean follow-up period of 52 months (median, 36 months; range, $<1-218$ months) and was comparable in all age groups. Of these 22 cases (Table 5), 21 (9\%) cases had high-risk features on histopathology and all but one patient received adjuvant systemic chemotherapy. One patient had no high-risk features in the enucleated eye but died due to non-compliance to treatment for the contralateral eye, which progressed to develop extraocular tumour extension subsequently. Seven patients had received additional external beam radiotherapy to the orbit. The most common high-risk features in patients who died due to RB were post-laminar optic nerve infiltration with/without involvement of optic nerve transection $(n=19)$ and massive choroidal infiltration $(n=17)$. All patients $(n=10)$ with involvement of optic nerve transection died due to central nervous system involvement.

\section{Discussion}

The incidence of high-risk RB varies from 19 to $66 \%$, with more cases in developing nations compared with the developed 
Table 3 High-risk retinoblastoma based on age at presentation: histopathology features

\begin{tabular}{|c|c|c|c|c|c|c|c|}
\hline Feature & $\begin{array}{l}\text { All ages } \\
(n=616)\end{array}$ & $\begin{array}{l}\text { Age }<1 \text { year } \\
(n=128)\end{array}$ & $\begin{array}{l}\text { Age } 1-2 \text { years } \\
(n=149)\end{array}$ & $\begin{array}{l}\text { Age } 2-3 \text { years } \\
(n=117)\end{array}$ & $\begin{array}{l}\text { Age } 3-4 \text { years } \\
(n=104)\end{array}$ & $\begin{array}{l}\text { Age }>4 \text { years } \\
(n=118)\end{array}$ & $p$ value \\
\hline \multicolumn{8}{|l|}{ Growth pattern $(n=610)$} \\
\hline Endophytic & $287(47)$ & $57(46)$ & $64(43)$ & $59(50)$ & $54(52)$ & $53(45)$ & 0.90 \\
\hline Exophytic & $241(40)$ & $59(47)$ & $68(46)$ & $42(36)$ & $33(32)$ & $39(33)$ & 0.36 \\
\hline Mixed & $63(10)$ & $7(6)$ & $13(9)$ & $14(12)$ & $11(11)$ & $18(15)$ & 0.21 \\
\hline Diffuse infiltrating & $18(3)$ & $2(2)$ & $3(2)$ & $2(2)$ & $6(6)$ & $5(4)$ & 0.27 \\
\hline \multicolumn{8}{|l|}{ Tumour differentiation $(n=615)$} \\
\hline Well-differentiated & $134(22)$ & $71(56)$ & $31(21)$ & $11(9)$ & $8(8)$ & $13(11)$ & $<0.001^{\circ}$ \\
\hline Moderate differentiation & $150(24)$ & $35(28)$ & $35(24)$ & $34(29)$ & $21(20)$ & $25(21)$ & 0.69 \\
\hline Poor differentiation & $304(49)$ & $19(15)$ & $75(51)$ & $68(58)$ & $67(65)$ & $75(64)$ & $<0.001^{\mathrm{t}}$ \\
\hline Undifferentiated & $25(4)$ & $2(2)$ & $7(5)$ & $4(3)$ & $7(7)$ & $5(4)$ & 0.42 \\
\hline Anterior chamber tumour seeds & $45(7)$ & $8(6)$ & $8(5)$ & $5(4)$ & $7(7)$ & $17(14)$ & 0.05 \\
\hline Iris infiltration & $28(5)$ & $2(2)$ & $5(3)$ & $4(3)$ & $6(6)$ & $11(9)$ & 0.06 \\
\hline Ciliary body infiltration & $29(5)$ & $4(3)$ & $5(3)$ & $2(2)$ & $7(7)$ & $11(9)$ & 0.06 \\
\hline Choroidal invasion & $186(30)$ & $31(24)$ & 49 (33) & $32(27)$ & $37(36)$ & $37(31)$ & 0.64 \\
\hline $\operatorname{Minor}(<3 \mathrm{~mm})$ & $66(11)$ & $19(15)$ & $14(9)$ & $10(9)$ & $8(8)$ & 15 (13) & 0.45 \\
\hline Massive $(\geq 3 \mathrm{~mm})$ & $120(19)$ & $12(9)$ & $35(23)$ & $22(19)$ & $29(28)$ & $22(19)$ & $0.04^{\mathrm{c}}$ \\
\hline Optic nerve infiltration & $279(45)$ & $51(40)$ & $74(50)$ & $55(47)$ & $47(45)$ & $52(44)$ & 0.89 \\
\hline Pre-laminar & $60(10)$ & $16(13)$ & $15(10)$ & $9(8)$ & $9(9)$ & $11(9)$ & 0.83 \\
\hline Laminar & $105(17)$ & $26(20)$ & $24(16)$ & $22(19)$ & $15(14)$ & $18(15)$ & 0.83 \\
\hline Post-laminar & $103(17)$ & $8(6)$ & $34(23)$ & $20(17)$ & $21(20)$ & $20(17)$ & $0.02^{\mathrm{d}}$ \\
\hline Optic nerve transection & $11(2)$ & $1(<1)$ & $1(<1)$ & $4(3)$ & $2(2)$ & $3(3)$ & 0.43 \\
\hline $\begin{array}{l}\text { Combination of pre-laminar/laminar optic } \\
\text { nerve and non-massive choroidal } \\
\text { involvement }\end{array}$ & $27(4)$ & $9(7)$ & $3(2)$ & $4(3)$ & $3(3)$ & $8(7)$ & 0.20 \\
\hline Scleral infiltration & $30(5)$ & $2(2)$ & $11(7)$ & $7(6)$ & $5(5)$ & $5(4)$ & 0.29 \\
\hline Extrascleral involvement & $10(2)$ & $1(<1)$ & $4(3)$ & $4(3)$ & $1(<1)$ & $0(0)$ & 0.20 \\
\hline High-risk features of retinoblastoma & $237(38)$ & $31(24)$ & $62(42)$ & $40(34)$ & $47(45)$ & $57(48)$ & 0.06 \\
\hline $\begin{array}{l}\text { Number of high-risk features Mean } \\
\text { (median, range) }\end{array}$ & $2(1,1-7)$ & $2(1,1-6)$ & $2(1,1-5)$ & $2(1,1-7)$ & $2(1,1-7)$ & $2(1,1-6)$ & 0.85 \\
\hline 1 & $150(24)$ & $23(18)$ & $40(27)$ & $24(21)$ & $29(28)$ & $34(29)$ & 0.42 \\
\hline 2 & $43(7)$ & $4(3)$ & $9(6)$ & $8(7)$ & $11(11)$ & $11(9)$ & 0.25 \\
\hline 3 & $24(4)$ & $2(2)$ & $6(4)$ & $3(3)$ & $3(3)$ & $10(8)$ & 0.08 \\
\hline 4 & $11(2)$ & $1(<1)$ & $5(3)$ & $4(3)$ & $1(<1)$ & $0(0)$ & 0.14 \\
\hline 5 & $5(<1)$ & $0(0)$ & $2(1)$ & $0(0)$ & $2(2)$ & $1(1)$ & 0.40 \\
\hline 6 & $2(<1)$ & $1(<1)$ & $0(0)$ & $0(0)$ & $0(0)$ & $1(1)$ & 0.56 \\
\hline 7 & $2(<1)$ & $0(0)$ & $0(0)$ & $1(<1)$ & $1(<1)$ & $0(0)$ & 0.47 \\
\hline
\end{tabular}

${ }^{a}$ Post-hoc analysis (Chi-square test with Bonferroni correction) showed that only age $\leq 1$ year was significantly different from all the other groups (all $p<0.001$ )

${ }^{\mathrm{b}}$ Post-hoc analysis (Chi-square test with Bonferroni correction) showed that only age $\leq 1$ year was significantly different from all the other groups (all $p<0.001)$

${ }^{c}$ Post-hoc analysis (Chi-square test with Bonferroni correction) showed that only age $\leq 1$ year was significantly different from ages $1-2$ years $(p=0.008)$ and ages $3-4$ years $(p=0.002)$

${ }^{\mathrm{d}}$ Post-hoc analysis (Chi-square test with Bonferroni correction) showed that only age $\leq 1$ year was significantly different from $1-2$ years $(p=0.001)$ and 3-4 years $(p=0.005)$

world [7]. In our study, the incidence of high-risk RB was $38 \%$. High-risk RB was more common in children aged more than 4 years (48\%) than children less than 1 year of age (24\%), though the findings were not statistically significant.
In a study of 297 primarily enucleated eyes of RB, Eagle Jr. noted that there was a statistically significant inverse relationship between the age at enucleation and the degree of tumour differentiation [15]. The mean age at enucleation for 
Table 4 High-risk retinoblastoma based on age at presentation: treatment and outcome

\begin{tabular}{|c|c|c|c|c|c|c|c|}
\hline Feature & $\begin{array}{l}\text { All ages } \\
(n=237)\end{array}$ & $\begin{array}{l}\text { Age }<1 \text { year } \\
(n=31)\end{array}$ & $\begin{array}{l}\text { Age } 1-2 \text { years } \\
(n=62)\end{array}$ & $\begin{array}{l}\text { Age } 2-3 \text { years } \\
(n=40)\end{array}$ & $\begin{array}{l}\text { Age 3-4 years } \\
(n=47)\end{array}$ & $\begin{array}{l}\text { Age }>4 \text { years } \\
(n=57)\end{array}$ & $p$ value \\
\hline Adjuvant systemic chemotherapy $(n=237)$ & $226(95)$ & $28(90)$ & $61(98)$ & $40(100)$ & $42(89)$ & $55(96)$ & 0.052 \\
\hline Systemic metastasis and death & $22(9)$ & $3(10)^{\mathrm{a}}$ & $4(6)$ & $6(15)$ & $4(9)$ & $5(9)$ & 0.89 \\
\hline
\end{tabular}

${ }^{a}$ One patient died due to non-compliance to treatment for the contralateral eye, which progressed to develop extraocular tumour extension. There were no high-risk features in the enucleated eye

Table 5 Details of patients who died due to retinoblastoma

\begin{tabular}{|c|c|c|c|c|c|}
\hline Case & Age group & $\begin{array}{l}\text { High-risk } \\
\text { retinoblastoma }\end{array}$ & Details of high-risk features & $\begin{array}{l}\text { Adjuvant } \\
\text { chemotherapy }\end{array}$ & $\begin{array}{l}\text { Adjuvant } \\
\text { radiotherapy }\end{array}$ \\
\hline 1 & $<1$ year & Yes & Massive choroidal invasion & Yes & No \\
\hline $2^{\mathrm{a}}$ & $<1$ year & No & N/A & N/A & N/A \\
\hline 3 & $<1$ year & Yes & $\begin{array}{l}\text { Anterior chamber seeding; ciliary body infiltration; massive } \\
\text { choroidal invasion; involvement of optic nerve transection; scleral } \\
\text { infiltration; extrascleral infiltration }\end{array}$ & Yes & No \\
\hline 4 & $1-2$ years & Yes & $\begin{array}{l}\text { Massive choroidal invasion; involvement of optic nerve } \\
\text { transection; scleral infiltration; extrascleral infiltration }\end{array}$ & Yes & Yes \\
\hline 5 & $1-2$ years & Yes & $\begin{array}{l}\text { Massive choroidal invasion; post-laminar optic nerve } \\
\text { infiltration; scleral infiltration; }\end{array}$ & Yes & No \\
\hline 6 & $1-2$ years & Yes & Massive choroidal invasion & Yes & No \\
\hline 7 & $1-2$ years & Yes & Massive choroidal invasion; post-laminar optic nerve infiltration & Yes & No \\
\hline 8 & $2-3$ years & Yes & $\begin{array}{l}\text { Massive choroidal invasion; post-laminar optic nerve } \\
\text { infiltration; scleral infiltration; extrascleral infiltration }\end{array}$ & Yes & No \\
\hline 9 & $2-3$ years & Yes & $\begin{array}{l}\text { Massive choroidal invasion; post-laminar optic nerve } \\
\text { infiltration; scleral infiltration; extrascleral infiltration }\end{array}$ & Yes & No \\
\hline 10 & $2-3$ years & Yes & $\begin{array}{l}\text { Massive choroidal invasion; involvement of optic nerve } \\
\text { transection; scleral infiltration }\end{array}$ & Yes & No \\
\hline 11 & $2-3$ years & Yes & $\begin{array}{l}\text { Massive choroidal invasion; involvement of optic nerve } \\
\text { transection; scleral infiltration; extrascleral infiltration }\end{array}$ & Yes & Yes \\
\hline 12 & $2-3$ years & Yes & $\begin{array}{l}\text { Anterior chamber seeding; iris infiltration; ciliary body } \\
\text { infiltration; massive choroidal invasion; involvement of optic } \\
\text { nerve transection; scleral infiltration; extrascleral infiltration }\end{array}$ & Yes & Yes \\
\hline 13 & $2-3$ years & Yes & Massive choroidal invasion; involvement of optic nerve transection & Yes & Yes \\
\hline 14 & $3-4$ years & Yes & Post-laminar optic nerve infiltration & Yes & No \\
\hline 15 & $3-4$ years & Yes & $\begin{array}{l}\text { Anterior chamber seeding; ciliary body infiltration; massive choroidal } \\
\text { invasion; involvement of optic nerve transection; scleral infiltration }\end{array}$ & Yes & No \\
\hline 16 & $3-4$ years & Yes & $\begin{array}{l}\text { Anterior chamber seeding; iris infiltration; ciliary body infiltration; } \\
\text { massive choroidal invasion; post-laminar optic nerve infiltration }\end{array}$ & Yes & No \\
\hline 17 & $3-4$ years & Yes & $\begin{array}{l}\text { Iris infiltration; ciliary body infiltration; massive choroidal } \\
\text { invasion; post-laminar optic nerve infiltration }\end{array}$ & Yes & No \\
\hline 18 & $>4$ years & Yes & Involvement of optic nerve transection & Yes & Yes \\
\hline 19 & $>4$ years & Yes & Massive choroidal invasion; post-laminar optic nerve infiltration & Yes & No \\
\hline 20 & $>4$ years & Yes & Involvement of optic nerve transection & Yes & Yes \\
\hline 21 & $>4$ years & Yes & Post-laminar optic nerve infiltration & No & No \\
\hline 22 & $>4$ years & Yes & Massive choroidal invasion; involvement of optic nerve transection & Yes & Yes \\
\hline
\end{tabular}

N/A not applicable

${ }^{a}$ One patient died due to non-compliance to treatment for the contralateral eye, which progressed to develop extraocular tumour extension. There were no high-risk features in the enucleated eye 
tumours with abundant rosettes was 10 months, moderate rosettes was 18 months, fewer rosettes was 20 months, and for poorly differentiated tumours was 34 months [15]. Similar findings have also been reported by Madhavan et al. in their study of 170 eyes with RB [16]. Younger patients were commonly associated with well-differentiated tumours while older patients were commonly associated with poorly differentiated tumours. Similarly, in our study, welldifferentiated tumours were more common in younger children $(56 \%$ in children $\leq 1$ year vs $11 \%$ in children older than 4 years; $p<0.001$ ) and poorly differentiated tumours were more common in older children $(15 \%$ in children $\leq 1$ year vs $64 \%$ in children older than 4 years; $p<0.001)$. This suggests that well-differentiated tumours present earlier than poorly differentiated tumours. It is speculated that this could be related to either retinal cell affection at different levels of development or may represent tumour progression with time and subsequent dedifferentiation of well-differentiated tumours into poorly differentiated tumours [17].

In our study, there was difference in the most common high-risk histopathological feature based on age at enucleation. Post-laminar optic nerve infiltration was least common in children $\leq 1$ year at $6 \%$ vs 17 to $23 \%$ in children older than 1 year, though the tumour basal diameter and thickness was comparable in all age groups. This finding could be related to the age-related changes in the biochemical composition of the extracellular matrix of lamina cribrosa, which is composed of collagen, elastin, and proteoglycans $[17,18]$. With increasing age, there is agerelated increase in total collagen and elastin, and decrease in type III to I collagen ratio and proteoglycan content $[17,18]$. This results in age-related decrease in the resilience of lamina cribrosa $[17,18]$. Better resilience to stress and pressure in very young children could be a protective factor against post-laminar optic nerve infiltration in children $\leq 1$ year and decreasing resilience with age could result in relatively easy access of tumour cells through the weak lamina cribrosa to post-laminar region in eyes with stress secondary to posterior pole exophytic tumours. However, there was no consistent increase in post-laminar optic nerve infiltration with advancing age. In our study, the occurrence of optic nerve infiltration decreased after the age of 2 years. This could be related to direct correlation between the patient age and location of tumour [19]. It is known that the tumours follow a central-to-peripheral distribution with advancing age, and most posterior pole tumours are detected before the age of 2 years [19].

Similarly, massive choroidal infiltration was least common in children $\leq 1$ year at $9 \%$ vs 19 to $28 \%$ in children older than 1 year in a setting of comparable tumour basal diameter and thickness in all age groups. Choroid is a vascular structure and is adherent to the sclera via numerous connective tissue strands, blood vessels, and nerves entering the choroid via the sclera. Thus scleral biochemical composition could influence the choroid behaviour. With increasing age, there is loss of collagen, elastin, and proteoglycans in the sclera resulting in a decrease in scleral elasticity [20]. High scleral elasticity and its influence on the overlying adherent choroid during very young age could be the cause of lesser chances of tumour infiltration to the underlying choroid.

Anterior chamber tumour seeding was more common in children older than 4 years at $14 \%$ compared with those $\leq 4$ years at $4-7 \%$. This finding could be related to increased chances of vitreous seeding in older children compared with younger children. In our study, vitreous seeding was more common in children $>4$ years $(53 \%)$ compared with children $\leq 4$ years $(21-34 \%)(p<0.0001)$. Free tumour cells in the vitreous or cell survival factors like VEGF and TGF- $\beta$ expressed by the tumour cells can spread via the aqueous humour between the ciliary epithelium and vitreous base into the anterior chamber via the pupil and proliferate, presenting as anterior chamber pseudohypopyon [21].

Based on age at enucleation, post-laminar optic nerve infiltration was common in children at 1-2 years of age, massive choroidal infiltration at 3-4 years, and anterior chamber tumour seeding, iris infiltration, and ciliary body infiltration in children $>4$ years of age. This could be related to the central-to-peripheral distribution of RB lesions with advancing age [19].

Previous studies have shown that $24 \%$ of untreated children with high-risk RB develop systemic metastasis and adjuvant systemic chemotherapy minimizes the risk of systemic metastasis and death to $0-4 \%$ in patients with high-risk RB [3, 4, 7, 22]. In our study, 9\% patients with high-risk RB died due to systemic or central nervous system metastasis, despite adjuvant systemic chemotherapy. This suggests that only $15 \%$ benefit from adjuvant systemic chemotherapy and the remaining $85 \%$ either receive unnecessary or ineffective treatment for high-risk RB. The most common high-risk feature associated with metastasis was post-laminar optic nerve infiltration, especially involvement of optic nerve transection, accounting for higher rate of systemic metastasis in this study. Despite aggressive treatment with chemotherapy and radiotherapy, involvement of optic nerve transection is associated with very high mortality of up to $80 \%$ [23-25]. Meticulous screening of optic nerve with pre-operative high-resolution magnetic resonance imaging of the orbit is mandatory prior to surgical planning.

In conclusion, the high-risk histopathology features vary based on age at enucleation. Earlier age at diagnosis and enucleation does not guarantee the absence of high-risk histopathology features, though the proportion of cases with high-risk features is less in younger children compared with older children. Optic nerve tumour infiltration and choroidal 
involvement is less common in children $\leq 1$ year of age compared with other age groups. This information is helpful in counselling the parents about the risk of high-risk features in eyes with advanced RB and need for further treatment based on age at diagnosis and/or enucleation.

\section{Summary}

\section{What was known before}

- High-risk features of retinoblastoma clinical features predicting high-risk retinoblastoma.

\section{What this study adds}

- The influence of age on the occurrence of high-risk retinoblastoma.

Acknowledgements Support provided by Operation Eyesight Institute for Eye Cancer (SK) and Hyderabad Eye Research Foundation (SK), Hyderabad, India. The funders had no role in the preparation, review, or approval of the manuscript.

\section{Compliance with ethical standards}

Conflict of interest The authors declare that they have no conflict of interest.

Publisher's note Springer Nature remains neutral with regard to jurisdictional claims in published maps and institutional affiliations.

\section{References}

1. Kaliki S, Patel A, Iram S, Ramappa G, Mohamed A, Palkonda VAR. Retinoblastoma in India: clinical presentation and outcome in 1457 Patients (2074 Eyes). Retina. 2019;39:379-91.

2. Fabian ID, Stacey AW, Chowdhury T, Duncan C, Karaa EK, Scheimberg I, et al. High-risk histopathology features in primary and secondary enucleated international intraocular retinoblastoma classification group D eyes. Ophthalmology. 2017;124:851-8.

3. Kaliki S, Shields CL, Shah SU, Eagle RC Jr, Shields JA, Leahey A. Postenucleation adjuvant chemotherapy with vincristine, etoposide, and carboplatin for the treatment of high-risk retinoblastoma. Arch Ophthalmol. 2011;129:1422-7.

4. Kaliki S, Srinivasan V, Gupta A, Mishra DK, Naik MN. Clinical features predictive of high-risk retinoblastoma in 403 Asian Indian patients: a case-control study. Ophthalmology. 2015;122:1165-72.

5. Sreelakshmi KV, Chandra A, Krishnakumar S, Natarajan V, Khetan V. Anterior chamber invasion in retinoblastoma: not an indication for adjuvant chemotherapy. Invest Ophthalmol Vis Sci. 2017;58:4654-61.
6. Chantada GL, Dunkel IJ, de Dávila MT, Abramson DH. Retinoblastoma patients with high risk ocular pathological features: who needs adjuvant therapy? Br J Ophthalmol. 2004; 88:1069-73.

7. Kaliki S, Shields CL, Eagle RC Jr, Iram S, Shields JA. High-risk intraocular retinoblastoma: comparison between Asian Indians and Americans from two major referral centers. Retina. 2018;38:2023-9.

8. Kivelä TT, Hadjistilianou T. Neonatal retinoblastoma. Asia Pac J Oncol Nurs. 2017;4:197-204.

9. Abramson DH, Du TT, Beaverson KL. (Neonatal) retinoblastoma in the first month of life. Arch Ophthalmol. 2002;120:738-42.

10. Abramson DH, Notterman RB, Ellsworth RM, Kitchin FD. Retinoblastoma treated in infants in the first six months of life. Arch Ophthalmol. 1983;101:1362-6.

11. Shields CL, Shields JA, Shah P. Retinoblastoma in older children. Ophthalmology 1991;98:395-9.

12. Reese AB, Ellsworth RM. The evaluation and current concept of retinoblastoma therapy. Trans Am Acad Ophthalmol Otolaryngol. 1963;67:164-72.

13. Shields CL, Mashayekhi A, Au AK, Czyz C, Leahey A, Meadows $\mathrm{AT}$, et al. The International Classification of Retinoblastoma predicts chemoreduction success. Ophthalmology. 2006; 113:2276-80.

14. Mallipatna AC, Gallie BL, Chévez-Barrios P, Lumbroso-Le Rouic L, Chantada G, et al. Retinoblastoma. In: Amin MB, Edge SB, Greene FL, Byrd DR, Brookland RK, et al., editors. AJCC Cancer Staging Manual. 8th ed. Switzerland: Springer; 2017. 819-31.

15. Eagle RC Jr. High-risk features and tumor differentiation in retinoblastoma: a retrospective histopathologic study. Arch Pathol Lab Med. 2009;133:1203-9.

16. Madhavan J, Ganesh A, Roy J, Biswas J, Kumaramanickavel G. The relationship between tumor cell differentiation and age at diagnosis in retinoblastoma. J Pediatr Ophthalmol Strabismus. 2008;45:22-5.

17. Albon J, Karwatowski WS, Avery N, Easty DL, Duance VC. Changes in the collagenous matrix of the aging human lamina cribrosa. Br J Ophthalmol. 1995;79:368-75.

18. Albon J, Purslow PP, Karwatowski WS, Easty DL. Age related compliance of the lamina cribrosa in human eyes. Br J Ophthalmol. 2000;84:318-23.

19. Abramson DH, Gombos DS. The topography of bilateral retinoblastoma lesions. Retina. 1996;16:232-9.

20. Rada JA, Achen VR, Penugonda S, Schmidt RW, Mount BA. Proteoglycan composition in the human sclera during growth and aging. Invest Ophthalmol Vis Sci. 2000;41:1639-48.

21. Crosby MB, Hubbard GB, Gallie BL, Grossniklaus HE. Anterior diffuse retinoblastoma: mutational analysis and immunofluorescence staining. Arch Pathol Lab Med. 2009; 133:1215-8

22. Honavar SG, Singh AD, Shields CL, Meadows AT, Demirci H, Cater J, et al. Postenucleation adjuvant therapy in high-risk retinoblastoma. Arch Ophthalmol. 2002;120:923-31.

23. Magramm I, Abramson DH, Ellsworth RM. Optic nerve involvement in retinoblastoma. Ophthalmology. 1989;96:217-22.

24. Shields CL, Shields JA, Baez K, Cater JR, De Potter P. Optic nerve invasion of retinoblastoma. Metastatic potential and clinical risk factors. Cancer. 1994;73:692-8.

25. Kaliki S, Tahiliani P, Mishra DK, Srinivasan V, Ali MH, Reddy VA. Optic nerve infiltration by retinoblastoma: predictive clinical features and outcome. Retina. 2016;36:1177-83. 